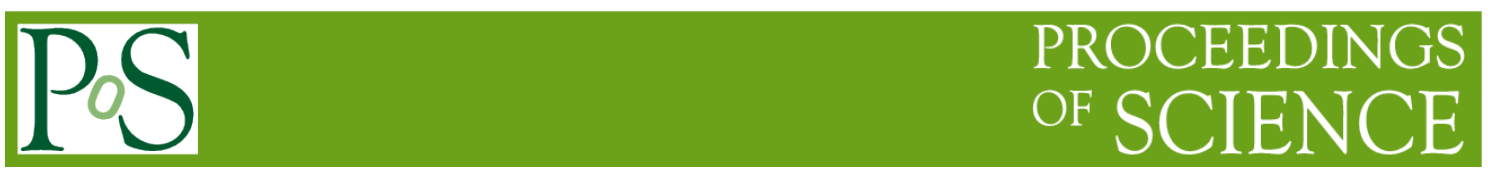

\title{
Pilot production of RPCs for the SHiP experiment
}

\author{
K. S. Lee ${ }^{1 *}$, \\ ${ }^{1}$ Dept. Of Physics and Institute of Basic Science, Korea University, Seoul 02841, Korea \\ E-mail:kslee0421@korea.ac.kr
}

M. Kang', Y. Jo', S. K. Park', S. H. Kim², J.-W. Ko², K. Y. Lee², B. D. Park², J. Y.

Sohn', C. S. Yoon ${ }^{2}$, J.-K. Woo', Y. G. Kim ${ }^{4}$, and K.-Y. Choi ${ }^{5}$

${ }^{2}$ Dept. of Physics Education \& RINS, Gyeongsang National University, Jinju 52828, Korea

${ }^{3}$ Dept. of Physics, Jeju National University, Jeju 63243, Korea

${ }^{4}$ Dept. of Science Education, Gwangju National University of Education, Gwangju 61204, Korea

${ }^{5}$ Dept. of Physics, Sungkyunkwan University, Suwon 16419, Korea

Two main aims of the SHiP (Search for Hidden Particles) experiment are the observation of hidden particles and high-statistics study of tau neutrino events. These particles can be produced from the decay of charmed particles in a SHiP hybrid target which is composed of a totally 58$\mathrm{cm}$ long series of TZM slabs followed by Tungsten slabs of the same total length. To achieve the physics goals, we carried out a test experiment with the SHiP target replica using CERN SPS $400 \mathrm{GeV} / \mathrm{c}$ proton beam at $\mathrm{H} 4$ area in July 2018. A major concern for the test experiment is the precise knowledge of the muon flux and the associated charm production cross section. In this test experiment, RPCs will be used for muon identification and their slope measurements. Recently, we have fabricated gas gaps and strip panels to build 5 trigger RPC modules for the present test experiment. In addition, we constructed a prototype RPC to study the fundamental detector performance using cosmic rays. The current construction of the RPC modules is also as a pilot production for the future SHiP experiment in synergy with the present RPC production for the CMS experiment.

The 39th International Conference on High Energy Physics (ICHEP2018)

4-11 July, 2018

Seoul, Korea

$1 *$ Speaker 


\section{Introduction}

The goals of the test experiment performed using the CERN SPS $400 \mathrm{GeV} / \mathrm{c}$ protons are to obtain the precise knowledge of the muon flux and the associated charm-production cross section at the beam energy of the SHiP experiment $[1,2]$.

\section{Trigger RPCs of the SHiP experiment}

A ShiP trigger RPC is composed of a 2-mm thick gas gap sandwiched by two orthogonal readout strips of a pitch of $10.625 \mathrm{~mm}$. Prior to the test experiment at CERN, we constructed and tested a prototype RPC of a size of $100 \times 130 \mathrm{~cm}^{2}$ to study the fundamental detector performance using cosmic rays.

\section{Cosmic-ray Test of the prototype RPC}

The left figure in Fig. 1 shows the conceptual design of the ShiP. The right figure shows muon efficiencies $\varepsilon_{\mu}$ and mean strip multiplicities $\left\langle C_{s}>\right.$ measured on $x$ (anode) and $y$ (cathode) strips of the SHiP prototype RPC as functions of effective high voltage $\left(\mathrm{HV}_{\text {eff }}\right)$ [3]. The working point (WP) $\mathrm{HV}_{\text {eff }}$ where $\varepsilon_{\mu}$ requiring both $x$ - and $y$-strip signals exceeds $95 \%$ is measured at $9.824 \mathrm{kV} .<C_{\mathrm{s}}>$ at the WP are measured to be about 1.7 for anode $(x)$ and about 2.3 for cathode (y) strips, respectively. The probability of large pulses defined as $C_{s}>6$ and the stochastic noise rate at the WP are on the order of $1.5 \%$ and $650 \mathrm{~Hz} \mathrm{~m}^{-2}$, respectively.

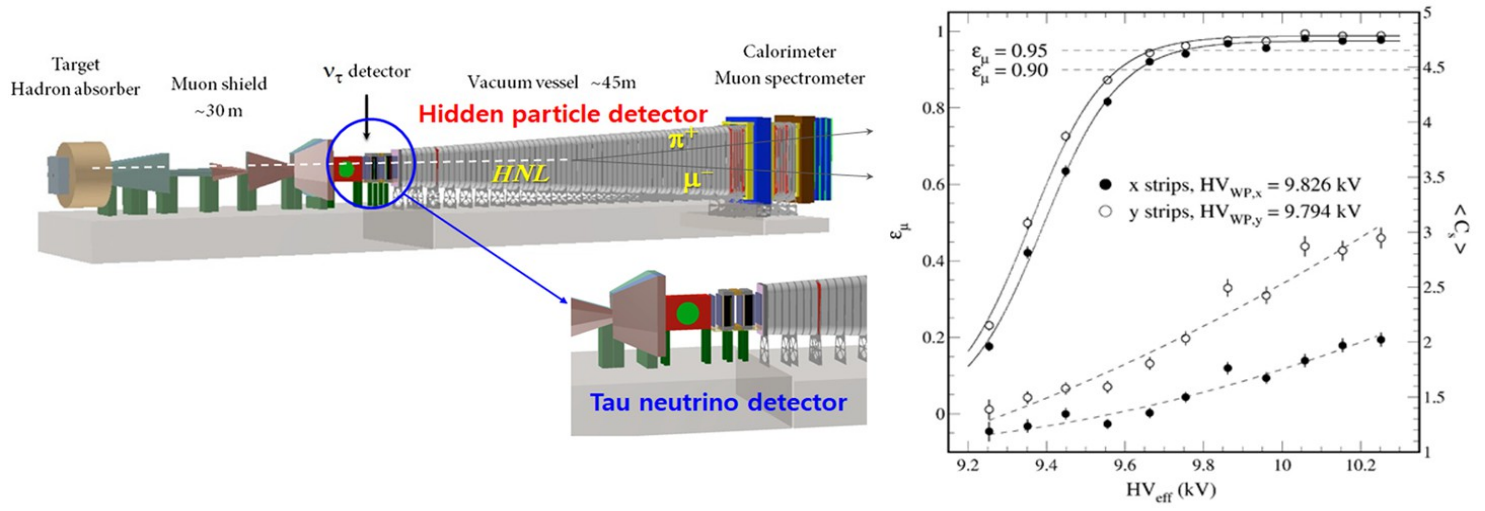

Figure 1. Conceptual design of the SHiP (left) and $\varepsilon_{\mu}$ and $\left\langle C_{s}>\right.$ measured on $x$ (anode) and $y$ (cathode) strips of the SHiP prototype RPC as functions of $\mathrm{HV}_{\text {eff }}$ (left).

\section{Conclusions}

The detector components, gaps and strips, have been manufactured, tested by the Korean SHiP group, and delivered to CERN for the pilot experiment at CERN SPS. The detector performance of the prototype RPC that appears in the present cosmic-muon test are fairly satisfactory for reliable 2D trigger measurements for the future SHiP.

\section{Acknowledgment}

This study was supported by the National Research Foundation of Korea with grant numbers of 2018r1a2b2007757, 2018r1d1a3b07050649, 2018r1d1a1b07050701, 2017r1d1a1b03036042, 2017r1a6a3a01075752, 2016r1a2b4012302, and 2016r1a6a3a11930680.

\section{References}

[1] G. DeLellis on behalf of the SHiP collaboration, JINST 11 C07003 (2016).

[2] 'SHiP: a new facility with a dedicated detector to search for new long-lived neutral particles', Elena Graverini on behalf of the SHiP collaboration, European Physical Society Conference on High Energy Physics, Vienna Austria, July 2015.

[3] K. S. Lee on behalf of the CMS collaboration, JINST 11 C08008 (2016). 